\title{
XRCC1 Gene Polymorphism, Clinicopathological Characteristics and Stomach Cancer Survival in Thailand
}

\author{
Nuntiput Putthanachote ${ }^{1,8}$, Supannee Promthet ${ }^{2 *}$, Krittika Suwanrungruan', \\ Peechanika Chopjitt ${ }^{4}$, Surapon Wiangnon ${ }^{5}$, Li-Sheng Chen ${ }^{6}$, Ming-Fang Yen ${ }^{6}$, \\ Tony Hsiu-Hsi Chen ${ }^{7}$
}

\begin{abstract}
Background: Stomach cancer is one of leading causes of death worldwide. In Thailand, the incidence and mortality of stomach cancer are in the top ten for cancers. Effects of DNA repair gene X-ray repair cross complementary protein 1 (XRCC1) polymorphisms and clinicopathological characteristics on survival of stomach cancer in Thailand have not been previously reported. The aim of this study was to investigate the effects of $X R C C 1$ gene and clinicopathological characteristics on survival of stomach cancer patients in Thailand. Materials and Methods: Data and blood samples were collected from 101 newly diagnosed stomach cancer cases pathologically confirmed and recruited during 2002 to 2006 and followed-up for vital status until 31 October 2012. Genotype analysis was performed using real-time PCR-HRM. The data were analyzed using the Kaplan-Meier method to yield cumulative survival curve, log-rank test to assess statistical difference of survival and Cox proportional hazard models to estimate adjusted hazard ratio. Results: The total followed-up times were 2,070 person-months, and the mortality rate was 4.3 per 100 person-months. The median survival time after diagnosis was 8.07 months. The cumulative 1-, 3-, 5-years survival rates were $40.4 \%, 15.2 \%$ and $10.1 \%$ respectively. After adjustment, tumour stage were associated with an increased risk of death $(\mathrm{p}=0.036)$. The XRCC1 GIn339Arg, Arg/Arg homozygote was also associated with increased risk but statistically this was non-significant. Conclusions: In addition to tumour stage, which is an important prognostic factor affecting to the survival of stomach cancer patients, the genetic variant Gln339Arg in $X R C C 1$ may non-significantly contribute to risk of stomach cancer death among Thai people. Larger studies with different populations are need to verify ours findings.
\end{abstract}

Keywords: XRCC1 polymorphism - survival - clinicopathological - stomach cancer - Thailand

Asian Pac J Cancer Prev, 16 (14), 6111-6116

\section{Introduction}

More than 990,000 cases of stomach cancer have been diagnosed and more than 738,000 deaths have occurred worldwide. The highest mortality rates were reported in Eastern Asia with a rate of 28.1 per 100,000 in males and 13.0 per 100,000 in females. The lowest mortality rates were reported in the Northern America (Ferlay et al., 2010). In Thailand, stomach cancer is one of the most common forms of malignancies. The overall estimated age-standardized incidence rate (ASR) for males was 4.5 per 100,000 for and 1.4 per 100,000 for females (Suwanrungruang et al., 2006).

The $\mathrm{X}$-ray repair cross-complementing group 1 $(X R C C 1)$ is one type of genetic variant that has been implicated in cancer susceptibility. From the evidence 297 case-control studies found XRCC1 Arg399Gln increases risk for overall cancer (Yi et al., 2013) and many studies suggest that the XRCC1 gene is one of the most important genetic risk factors for stomach cancer (Hong et al., 2009; Engin et al., 2011; Yuan et al., 2011; Chen et., 2012; Pan et al., 2012; Qiao et al., 2013) and previous studies have pointed to $X R C C 1$ polymorphism as an important prognostic factor for survival of gastric cancer (Shim et al., 2010; Tahara et al., 2011; Deng et al., 2014; Zhang et al., 2014).

In terms of clinicopathological characteristics, Previously studies have reported tumor site, tumor size, lymph node ratio, staging of diseases, lymph node metastasis, tumor invasion, distant of metastases, Borrmann type, depth of invasion and surgical margin status all related to survival of stomach cancer patients (Yin

${ }^{1}$ Department of Epidemiology, Faculty of Public Health, ${ }^{2}$ Cancer Unit, ${ }^{3}$ Department of Microbiology, ${ }^{4}$ Department of Pediatrics, Faculty of Medicine, Khon Kaen University, Khon Kaen, ${ }^{7}$ Clinical Microbiology Laboratory, Roi-et Hospital, Roi-et Province, Thailand, ${ }^{5}$ College of Oral Medicine School of Oral Hygiene, Taipei Medical University, ${ }^{6}$ Institute of Epidemiology and Prevention Medicine, College of Public Health, National Taiwan University, Taiwan*For correspondence: supannee@kku.ac.th 
Nuntiput Putthanachote et al

et al., 2012; Li et al., 2013; Okholm et al., 2014; Posteraro et al., 2014; Deng et al., 2015). However, few studies have conducted in Thailand and none have been investigated the effects of clinicopathological characteristics or XRCCI polymorphism on survival of stomach cancer patients. It is quite possible that genetic and lifestyle differences between Thai and other population (particularly western population). This study investigates the effects of XRCC1 gene and clinicopathological characteristics on survival of stomach cancer patients in Thailand.

\section{Materials and Methods}

\section{Study subjects}

In total, 101 newly diagnosis stomach cancer patients were included in this study. All cases were histologically confirmed and diagnosed according to the International Classification of Diseases for Oncology (ICD-O 3rd). Subjects were recruited from Srinagarind Hospital and Khon Kaen Regional Hospital, Khon Kaen Province, Northeast Thailand, during 2002 to 2006 . All of patients were followed-up until death or the end of the study (31 October, 2012). Factors of interest were retrieved from medical records including age at diagnosis, gender, site of diseases, surgery type, histological type, histological grading and stage of disease. XRCCl genotyping was the performed (described below). The classical endpoint in this study is survival time of stomach cancer. Status of each patient was checked from medical records and by linkage with the death registry of the Thai national statistics database.

\section{Laboratory methods}

Specimen collection and DNA extraction: Blood samples were taken from all stomach cancer patients diagnosis in the study period $(n=101)$. Whole blood samples of 3-5 ml were collected and centrifuged at 3,000 rpm for 15 minute to separate plasma, buffy coat and red blood cells. All specimens were stored at $-20^{\circ} \mathrm{C}$ at the cancer unit, Faculty of Medicine, Khon Kaen University. The genomic DNA was extracted from the buffy coat at Nagoya city university medical school, Japan.

\section{PCR amplification and genetic polymorphisms detection}

The DNA analyses were performed by using real-time polymerase chain reaction with high resolution melting technique (Real-time PCR-HRM). DNA amplification was performed in a 96-well plate in the light $\mathrm{Cycler}{ }^{\circledR}$ 480 Real-Time PCR System. The amplification of XRCC1 Gln399Arg gene was used two primers, [Forward]: 5'AGT GGG TGC TGG ACT GTC-3' and [Reverse]:5'TTG CCC AGC ACA GGA TAA-3’. The HRM data were analyzed using the light Cycler ${ }^{\circledR} 480$ Gene Scanning software version 1.5 (Roche) and was performed at Department of Microbiology, Faculty of Medicine, Khon Kaen University.

\section{Statistical analysis}

Survival times of patient were calculated for each patient and were started from the date of diagnosis until the date of death or the end of follow-up (31 October,
2012). Percentages were used to describe categorical data and means with standard deviations or medians with ranges were used to describe continuous data. The observed survival rate was calculated and summarized using Kaplan-meier survival curves. The statistics used to compare survival between groups was performed by using the log-rank test. The univariate and multivariate Cox proportional hazard regression models were used to estimate the association between explanatory variable and survival experience, presented crude hazard ratios (HR) and adjusted HRs and their $95 \%$ confidence interval (CI). All analysis was conducted using the SAS statistical package (version 9.3; SAS institute, Cary, NC) and significance level of 0.05 was used for all analysis.

\section{The Ethics Consideration}

The study was approved by the Khon Kaen University Ethics Committee for Human Research. The reference number is HE561259.

\section{Results}

\section{Demographic characteristics of stomach cancer}

The results of the descriptive analysis were summarized in Table 1. Of the 101 patients with stomach cancer, 57 $(56.4 \%)$ were males. The mean age was 52.7 years. Most of the cancer patients were married $78.2 \%$, had only a primary school education were $74.3 \%$ and farmers or agricultural worker were $69.3 \%$. Table 2 shows the frequencies and the contribution of pathological characteristics of cases. The most commonly specified anatomical sites of stomach cancer were the antrum (45.6\% of all cases) and the cardia (16.8\% of all cases). The most common type of surgery was subtotal gastrectomy (49.5\%). Regarding histopathology, the most frequently specified histological type of malignancy was signet ring cells carcinoma (24.7\% of all cases), and in most patients histological grade was assessed as poorly differentiated $(58.4 \%)$ or unable to be assessed $(28.8 \%)$. Stage IV cancers $(53.5 \%)$ preponderated the majority of the patients. The allele frequencies of XRCC1 Gln399Arg polymorphisms for Gln/Gln, Gln/Arg and Arg/Arg genotypes were $47.5 \%, 40.6 \%$ and $11.9 \%$, respectively.

\section{Survival rate of stomach cancer}

The total follow-up person time was 2,070 personmonths, and the overall mortality rate was 4.3 per 100 person-months (95\%CI: 3.49 to 5.35). Table 3 presents the survival rates. The cumulative 3-, 6- and 9 months, 1 -, 3 - and 5-years survival rates were $86.9 \%, 63.7 \%, 46.5 \%$ $40.4 \%, 15.2 \%$ and $10.1 \%$, respectively. The median survival time of stomach cancer after diagnosis was 8.07 months (95\%CI: 6.00 to 10.23; Figure 1). The Figures 2-5 presented survival times of stage of diseases, histology type, histology grading and $X R C C 1$ polymorphisms. The median survival time of Stage IB, Stage II, Stage IIIA, Stage IIIB, Stage IV and Unknown stage were 9.10, 41.80, $22.90,14.14,8.67,6.27$ months respectively. Regarding to XRCC1 polymorphisms, the median survival time of Arg/ Arg, Gln/Arg and Gln/Gln genotype were 15.60, 12.30 and 7.33 months respectively. 
XRCC1 Gene Polymorphism, and Stomach Cancer Survival in Thailand

Table 1. The General Characteristics of Stomach Cancer

\begin{tabular}{lcc}
\hline Variables & Nunber(101) & $\%$ \\
\hline Gender & & \\
$\quad$ Male & 57 & 56.4 \\
$\quad$ Female & 44 & 43.6 \\
Age (years) & & \\
$\quad<60$ & 70 & 69.3 \\
$\quad$ 60 & 31 & 30.7 \\
Mean + - SD & $52.7(+-11.42)$ & \\
Median (Min:Max) & 53 & \\
Marital status & & \\
$\quad$ Single & $68: 70)$ & 5.9 \\
$\quad$ Married & 79 & 78.2 \\
$\quad$ Separated, widowed & 16 & 15.9 \\
Occupation & & \\
$\quad$ Agriculture, farmer & 70 & 69.3 \\
$\quad$ Office, technical work & 18 & 17.8 \\
$\quad$ Professional work & 13 & 12.9 \\
Education & & \\
$\quad$ Illiteracy & 2 & 2 \\
$\quad$ Primary school & 75 & 74.3 \\
$\quad$ Secondary school or higher & 24 & 23.7 \\
\hline
\end{tabular}

Table 2. Frequencies and Distribution of Pathological Characteristics of Stomach Cancer

\begin{tabular}{|c|c|c|}
\hline Variables & Nunber(101) & $\%$ \\
\hline \multicolumn{3}{|l|}{ Site of diseases } \\
\hline Fundus & 1 & 1.0 \\
\hline Pylorus & 2 & 2.0 \\
\hline Body & 7 & 6.9 \\
\hline Cardia & 17 & 16.8 \\
\hline Antrum & 46 & 45.6 \\
\hline Stomach, Not otherwise specified & 28 & 27.2 \\
\hline \multicolumn{3}{|l|}{ Type of surgery } \\
\hline Gastric mucosa biopsy & 21 & 21.0 \\
\hline Subtotal gastrectomy & 50 & 49.5 \\
\hline Near totalgastrectomy & 7 & 6.9 \\
\hline Total gastrectomy & 15 & 14.5 \\
\hline Orther & 8 & 8.1 \\
\hline \multicolumn{3}{|l|}{ Histology type } \\
\hline Tubular adenocarcinoma & 1 & 1.0 \\
\hline Diffuse type & 5 & 5.0 \\
\hline Signet ring cell carcinoma & 25 & 24.7 \\
\hline Adenocarcinoma, Not otherwise specified & 69 & 69.3 \\
\hline \multicolumn{3}{|l|}{ Histology Grading } \\
\hline Well differentiated & 10 & 9.9 \\
\hline Moderately differentiated & 11 & 10.9 \\
\hline Poorly differentiated & 59 & 58.4 \\
\hline Grade can't be assessed & 21 & 28.8 \\
\hline \multicolumn{3}{|l|}{ Stage of diseases } \\
\hline Stage IB & 3 & 3.0 \\
\hline Stage II & 5 & 5.0 \\
\hline Stage IIIA & 9 & 8.9 \\
\hline Stage IIIB & 6 & 5.9 \\
\hline Stage IV & 54 & 53.5 \\
\hline Unknown Stage & 24 & 23.8 \\
\hline \multicolumn{3}{|l|}{ XRCC1 G339A genotype } \\
\hline $\mathrm{Gln} / \mathrm{Gln}$ & 48 & 47.5 \\
\hline Gln/Arg & 41 & 40.6 \\
\hline Arg/Arg & 12 & 11.9 \\
\hline
\end{tabular}

The associated of Clinicopathological and XRCC1 gene Polymorphisms with survival of stomach cancer

Table 4 shows after adjusting for lymph node metastasis, comorbidity and complication. Tumour stage IV and Unknown stage lead to increased risked of death (HR: 3.6 ; $95 \%$ CI: 1.35 to 9.43 ; HR: 3.0 ; 95\%CI: 1.08

Table 3. Survival Rate of Stomach Cancer After Diagnosis

\begin{tabular}{lcccc}
\hline $\begin{array}{l}\text { Survival time } \\
\text { time (Months) }\end{array}$ & $\begin{array}{c}\text { Median } \\
\text { (M) CI }\end{array}$ & $\begin{array}{c}\text { Survival } \\
\text { rate (\%) }\end{array}$ & $95 \%$ CI \\
\hline 3 Months & 1.9 & $0.63-2.07$ & 86.9 & $78.49-92.17$ \\
6 Months & 3.5 & $2.80-4.60$ & 63.7 & $53.36-72.27$ \\
9 months & 5.1 & $4.00-5.70$ & 46.5 & $36.43-55.90$ \\
1 Year & 5.5 & $4.40-6.07$ & 40.4 & $30.73-49.87$ \\
3 Years & 6.9 & $5.70-8.73$ & 15.2 & $8.93-22.90$ \\
5 Years & 7.8 & $5.80-10.23$ & 10.1 & $5.17-16.97$ \\
\hline
\end{tabular}

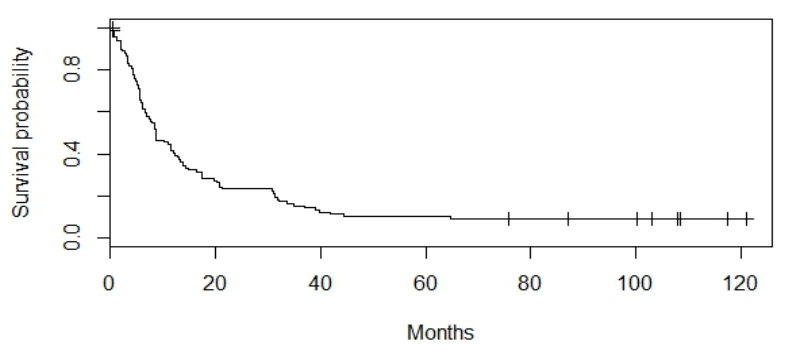

Figure 1. Overall Survival Curve of Stomach Cancer

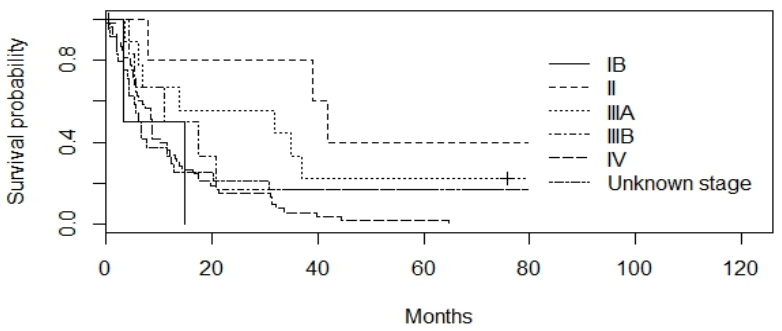

Figure 2. Survival Curve of Stomach Cancer by Stage of Diseases

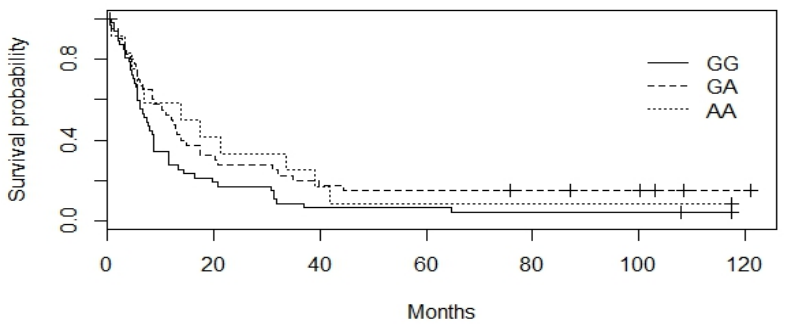

Figure 3. Survival Curve of Stomach Cancer by XRCC1 Gene

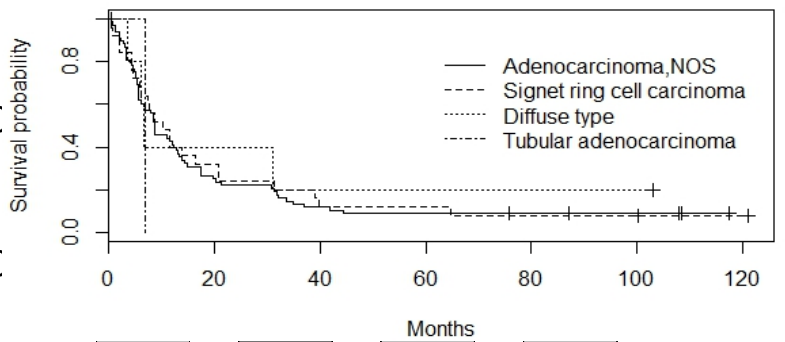

Figure 4. Survival Curve of Stomach Cancer by Histology Type 
Table 4. Pathological and XRCC1 Gene as Effected to Survival of Stomach Cancer (Multivariate Analysis)

\begin{tabular}{|c|c|c|c|c|c|}
\hline Variable & Number(\%) & $\begin{array}{c}\text { Median time } \\
\text { (Months) 95\% CI }\end{array}$ & $\begin{array}{l}\text { Crude HR } \\
(95 \% \mathrm{CI})\end{array}$ & $\begin{array}{l}\text { Adjusted HR } \\
(95 \% \mathrm{CI})\end{array}$ & $\overline{\mathrm{p} \text {-value }}$ \\
\hline Gender & & & & & 0.306 \\
\hline Male & $57(56.4)$ & $10.3(6.1-14.3)$ & 1 & 1 & \\
\hline Female & $44(43.6)$ & $8.1(5.7-12.3)$ & $1.2(0.81-1.87)$ & $1.2(0.81-1.89)$ & \\
\hline Age & & & & & 0.414 \\
\hline$<60$ & $70(69.3)$ & $8.6(6.7-12.3)$ & 1 & 1 & \\
\hline$>60$ & $31(30.7)$ & $10.1(4.8-17.4)$ & $0.9(0.62-1.49)$ & $1.0(0.59-1.44)$ & \\
\hline Site of diseases & & & & & 0.308 \\
\hline Fundus, Pylorus, Body & $10(10.4)$ & $13.7(5.1-20.2)$ & 1 & 1 & \\
\hline Cardia & $17(16.8)$ & $12.8(3.4-20.2)$ & $2.2(0.87-5.65)$ & $2.3(0.90-6.08)$ & \\
\hline Antrum & $46(45.6)$ & 7.8(5.6-11.9) & $2.0(0.86-4.81)$ & $2.1(0.85-5.04)$ & \\
\hline Stomach, NOS & $28(27.2)$ & $8.4(5.5-12.3)$ & $2.1(0.89-5.31)$ & $2.4(0.95-6.02)$ & \\
\hline Type of surgery & & & & & 0.149 \\
\hline Gastric mucosa biopsy & $21(21.0)$ & $6.5(2.3-12.3)$ & 1 & 1 & \\
\hline Subtotal gastrectomy & $50(49.5)$ & $11.6(6.1-17.3)$ & $0.4(0.05-2.65)$ & $0.3(0.04-3.05)$ & \\
\hline Near totalgastrectomy & $7(6.9)$ & $11.9(2.8-64.6)$ & $0.3(0.03-2.47)$ & $0.2(0.02-2.10)$ & \\
\hline Total gastrectomy & $15(14.5)$ & $11.5(1.9-31.4)$ & $0.3(0.04-2.45)$ & $0.3(0.04-2.73)$ & \\
\hline Orther & $8(8.1)$ & $4.5(1.2-7.8)$ & $1.2(0.14-9.45)$ & $1.3(0.15-11.85)$ & \\
\hline \multicolumn{6}{|l|}{ Histology type } \\
\hline Tubular adenocarcinoma, Diffuse type & $6(6.0)$ & $6.7(3.5-\mathrm{NA})$ & 1 & 1 & 0.657 \\
\hline Signet ring cell carcinoma & $25(24.7)$ & $10.2(5.8-20.6)$ & $0.6(0.08-4.60)$ & $0.7(0.08-5.54)$ & \\
\hline Adenocarcinoma, NOS & $69(69.3)$ & $8.7(5.7-12.9)$ & $0.7(0.09-4.72)$ & $0.9(0.11-6.81)$ & \\
\hline Histology grading & & & & & 0.638 \\
\hline Well differentiated & $10(9.9)$ & $6.8(2.3-31.4)$ & 1 & 1 & \\
\hline Moderately differentiated & $11(10.9)$ & $12.8(5.7-21.3)$ & $0.8(0.45-1.63)$ & $0.7(0.27-1.88)$ & \\
\hline Poorly differentiated & $59(58.4)$ & $8.7(6.7-13.0)$ & $1.3(0.77-1.79)$ & $0.8(0.37-1.70)$ & \\
\hline Grade can't be assessed & $21(28.8)$ & $6.1(3.5-14.8)$ & $0.8(0.46-1.37)$ & $0.6(0.25-1.54)$ & \\
\hline Stage of diseases & & & & & 0.036 \\
\hline Stage IB+II & 8(7.9) & 39.1( 3.4-NA) & 1 & 1 & \\
\hline Stage IIIA+IIIB & $15(14.8)$ & $15.6(5.4-35.1)$ & $1.4(0.49-4.15)$ & $1.9(0.63-5.71)$ & \\
\hline Stage IV & $54(53.5)$ & $8.7(5.8-11.5)$ & $2.8(1.09-7.02)$ & $3.6(1.35-9.43)$ & \\
\hline Unknown Stage & $24(23.8)$ & $6.2(4.0-12.3)$ & $2.3(1.87-6.21)$ & $3.0(1.08-8.34)$ & \\
\hline XRCC1 G339A genotype & & & & & 0.136 \\
\hline $\mathrm{G} \ln / \mathrm{Gln}$ & $48(47.5)$ & $7.3(5.5-8.7)$ & 1 & 1 & \\
\hline Gln/Arg & $41(40.6)$ & $12.3(6.5-17.5)$ & $0.6(0.40-0.98)$ & $1.0(0.52-2.02)$ & \\
\hline Arg/Arg & 12(11.9) & $15.6(3.3-39.1)$ & $1.6(1.02-2.50)$ & $1.8(0.89-3.45)$ & \\
\hline
\end{tabular}

*Stomach cancer; 95\% CI, 95\% confidence interval, were adjusted for complication, comorbidity and metastasis using Cox proportional hazard regression models, p-value from Partial likelihood ratio test; NA, Not Applicable; NOS, not otherwise specified, Other = Gastrojejunectomy, Hemi gastrectomy and Esophagogastrectomy

Table 5. Final Multivariate Model of Significant Factors Independently Associated with Hazard of Death

\begin{tabular}{lccc}
\hline Variable & HRa & $95 \%$ CI & p-value \\
\hline Stage IV & 1.7 & $1.09-2.59$ & 0.019 \\
\hline
\end{tabular}

*Stomach cancer; HRa, Adjusted Hazard Ratio; 95\% CI, 95\% confidence interval p-value base on stepwise cox proportional hazards regression

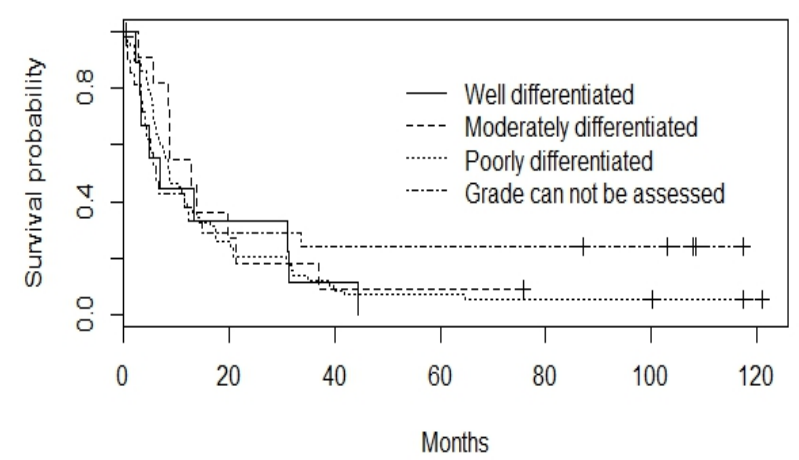

Figure 5. Survival Curve of Stomach Cancer by Histology Grading to 8.34). The Polymorphisms of XRCC1 Gln339Arg were associated increased risked of death with Arg/ Arg homozygote but we can't demonstrated statistically significant. Table 5 show the final multivariate model of significant factors independently associated with hazard of death base on stepwise Cox proportional hazards regression and found a tumour stage IV was associated with hazard of death 1.7-fold (95\%CI: 1.09 to 2.59).

\section{Discussion}

Our study investigated the factors associated with mortality among stomach cancer patients. This is firstly reported on the effected of the XRCC1 gene and clinicopathological characteristics on the survival of stomach cancer patients among Thai peoples. Our resulted found the stage of diseases was the factors affected to survival of patients, which is consistent with previous studies have been reported. They found out that the staging of diseases were impotent factors affected to survival of gastric cancer patients especially advance stage of diseases (Choi et al., 2011; Kwon et al., 2014). 
The study on the effected of the XRCCl gene to survival of stomach cancer patients, many studies have been explored much on the associated of XRCC1 gene and clinical outcome to survival of patients under treatment by chemotherapy (Liu et al., 2007; Wang et al., 2012; Zou and Yang, 2012; Xu et al., 2014) but non studies have been conducted in Thailand. Our results have found out that the XRCC1 Gln399Arg, Arg/Arg homozygote was affected to survival of stomach cancer patients but statistically non significant.

The tumor location and type of surgeries has important factors affected to survival of stomach cancer patients. Ours study found the tumor location and type of surgeries were not increases risked of death. This is inconsistent with preciously studies done in Korea, France and China they found out that the location of cancer in the stomach and type of surgeries were important factor that effected to survival of stomach cancer patients (Choi et al., 2011; Deng et al., 2014; Son et al., 2014; Herbreteau et al., 2015). The histology grading and histology type, our study found not increases risked of death, Similar findings have been previously reported elsewhere (Kwon et al., 2014; Son et al., 2014).

In conclusion, our study suggests the stage of diseases is the factors affecting to survival of stomach cancer in Thai population. We did not find any effects of $X R C C 1$ polymorphisms, tumor location, surgeries type, histology grading and histology type were associated with an increase risk of death of stomach cancer patients. It would be necessary to confirm these findings in the larger sample size.

\section{Acknowledgements}

The authors also would like to thank staff from National Taiwan University and Taipei Medical University, Taiwan for training N. Putthanachote in data analysis and writing this manuscript.

Thanks are also due to MONKASHO for its initial support at the first phase of the project. We wish to acknowledge Professor Tokudome for initiating the International collaborative epidemiological study. Finally, Thanks are due to Dr.Cameron Hurst for his advice and assistance in writing this paper.

\section{References}

Chen B, Zhou Y,Yang P, et al (2012). Polymorphisms of XRCC1 and gastric cancer susceptibility: A meta-analysis. Molecular Biology Reports, 39, 1305-13.

Choi JY,Shim KN, Roh SH, et al (2011). [Clinicopathological characteristics of gastric cancer and survival improvement by surgical treatment in the elderly]. The Korean journal of gastroenterology = Taehan Sohwagi Hakhoe chi, 58, 9-19.

Deng J, Zhang R, Pan Y, et al (2015). Tumor size as a recommendable variable for accuracy of the prognostic prediction of gastric cancer: a retrospective analysis of 1,521 patients. Annals of Surgical Oncology, 22, 565-72.

Deng J, Zhang R, Pan Y, et al (2014). N stages of the seventh edition of TNM Classification are the most intensive variables for predictions of the overall survival of gastric cancer patients who underwent limited lymphadenectomy.
Tumor Biology, 35, 3269-81.

Engin AB, Karahalil B, Karakaya A, Engin A (2011). Association between XRCC1 ARG399GLN and P53 ARG72PRO polymorphisms and the risk of gastric and colorectal cancer in Turkish population. Arhiv Za Higijenu Rada I Toksikologiju, 62, 207-14.

Ferlay J, Shin HR, Bray F, et al (2010). Estimates of worldwide burden of cancer in 2008: GLOBOCAN 2008. International Journal of Cancer, 127, 2893-917.

Herbreteau E, Jooste V, Hamza S, Lepage C, et al (2015). Trends in the management of gastric cancer over a 32-year period: a French population-based study. Gastric Cancer: Official Journal of the International Gastric Cancer Association and the Japanese Gastric Cancer Association, 18, 129-37.

Hong SH, Choi JK, Ahn DH, et al (2009).Association of the $X R C C 1$ gene polymorphisms in patients with stomach cancer. Genes and Genomics, 31, 435-41.

Kwon KJ, Shim KN, Song EM , et al (2014). Clinicopathological characteristics and prognosis of signet ring cell carcinoma of the stomach. Gastric Cancer, 17, 43-53.

Li F, Zhang R, Liang H, et al (2013). A retrospective clinicopathologic study of remnant gastric cancer after distal gastrectomy. American Journal of Clinical Oncology: Cancer Clinical Trials, 36, 244-9.

Liu B, Wei J, Zou Z, et al (2007). Polymorphism of XRCC1 predicts overall survival of gastric cancer patients receiving oxaliplatin-based chemotherapy in Chinese population. European Journal of Human Genetics, 15, 1049-53.

Okholm C, Svendsen LB, Achiam MP (2014). Status and prognosis of lymph node metastasis in patients with cardia cancer - a systematic review. Surgical Oncology, 23, 140-6.

Pan XF, Xie Y, Loh M, et al (2012). Polymorphisms of XRCC1 and ADPRT genes and risk of noncardia gastric cancer in a Chinese population: A casecontrol study. Asian Pac J Cancer Prev, 13, 5637-42.

Posteraro B, Persiani R, Dall'Armi V, et al (2014). Prognostic factors and outcomes in Italian patients undergoing curative gastric cancer surgery. European Journal of Surgical Oncology, 40, 345-51.

Qiao W, Wang T, Zhang L, et al (2013). Association study of single nucleotide polymorphisms in XRCCl gene with the risk of gastric cancer in Chinese population. International Journal of Biological Sciences, 9, 753-8.

Shim HJ, Yun JY, Hwang JE, et al (2010). BRCA1 and XRCC1 polymorphisms associated with survival in advanced gastric cancer treated with taxane and cisplatin. Cancer Science, 101, 1247-54.

Son T, Hyung WJ, Kim JW, et al (2014). Anatomic extent of metastatic lymph nodes: Still important for gastric cancer prognosis. Annals of Surgical Oncology, 21, 899-7.

Suwanrungruang K, Wiangnon S, Sriamporn S, et al (2006). Trends in incidences of stomach and colorectal cancer in Khon Kaen, Thailand 1985-2004. Asian Pac J Cancer Prev, 7, 623-6.

Tahara T, Shibata T, Nakamura M, et al (2011). Effect of genetic polymorphisms related to DNA repair and the xenobiotic pathway on the prognosis and survival of gastric cancer patients. Anticancer Research, 31, 705-10.

Wang S, Wu X, Chen Y, et al (2012). Prognostic and predictive role of JWA and XRCC1 expressions in gastric cancer. Clinical Cancer Research, 18, 2987-96.

$\mathrm{Xu} \mathrm{J,} \mathrm{Ma} \mathrm{J,} \mathrm{Zong} \mathrm{HT,} \mathrm{et} \mathrm{al} \mathrm{(2014).} \mathrm{Pharmacogenetic} \mathrm{role}$ of XRCC1 polymorphisms on the clinical outcome of gastric cancer patients with platinum-based chemotherapy: A systematic review and meta-analysis. Genetics and Molecular Research, 13, 1438-46.

Yi L, Xiao-Feng H, Yun-Tao L, et al (2013). Association between 
Nuntiput Putthanachote et al

the XRCC1 Arg399Gln polymorphism and risk of cancer: evidence from 297 case-control studies. PloS One, 8, 78071.

Yin C, Li D, Sun Z, et al (2012). Clinicopathologic features and prognosis analysis of mucinous gastric carcinoma. Medical Oncology, 29, 864-70.

Yuan T, Deng S, Chen M, et al (2011). Association of DNA repair gene $X R C C 1$ and XPD polymorphisms with genetic susceptibility to gastric cancer in a Chinese population. Cancer Epidemiology, 35, 170-4.

Zhang X, Jiang LP, Yin Y, Wang YD. (2014). XRCC1 and XPD genetic polymorphisms and clinical outcomes of gastric cancer patients treated with oxaliplatin-based chemotherapy: a meta-analysis. Tumour Biology: The Journal of the International Society for Oncodevelopmental Biology and Medicine, 35, 5637-45.

Zou HZ, Yang SJ (2012). Prediction role of seven SNPs of DNA repair genes for survival of gastric cancer patients receiving chemotherapy. Asian Pac J Cancer Prev, 13, 6187-90. 